\title{
Topical Application of Ketoprofen Improves Gait Disturbance in Rat Models of Acute Inflammation
}

\author{
Yosuke Amagai, ${ }^{1}$ Akane Tanaka, ${ }^{1,2}$ Akira Matsuda, ${ }^{3}$ Kumiko Oida, ${ }^{1}$ Kyungsook Jung, \\ Sho Nishikawa, ${ }^{1}$ Hyosun Jang, ${ }^{1}$ Saori Ishizaka, ${ }^{1}$ and Hiroshi Matsuda ${ }^{1,3}$ \\ ${ }^{1}$ Cooperative Major in Advanced Health Science, Graduate School of Bio-Applications and System Engineering, \\ Tokyo University of Agriculture and Technology, 3-8-5 Saiwai-cho, Fuchu, Tokyo, Japan \\ ${ }^{2}$ Laboratories of Comparative Animal Medicine, Tokyo University of Agriculture and Technology, 3-8-5 Saiwai-cho, Fuchu, \\ Tokyo, Japan \\ ${ }^{3}$ Veterinary Molecular Pathology and Therapeutics, Division of Animal Life Science, Tokyo University of Agriculture and Technology, \\ 3-8-5 Saiwai-cho, Fuchu, Tokyo, Japan
}

Correspondence should be addressed to Akane Tanaka; akane@cc.tuat.ac.jp and Hiroshi Matsuda; hiro@cc.tuat.ac.jp

Received 4 April 2013; Accepted 11 July 2013

Academic Editor: Todd Vanderah

Copyright (c) 2013 Yosuke Amagai et al. This is an open access article distributed under the Creative Commons Attribution License, which permits unrestricted use, distribution, and reproduction in any medium, provided the original work is properly cited.

Arthritis is a disabling health problem and commonly develops in the late stages of life; the condition is typically accompanied by chronic pain. For the assessment of pain severity and therapeutic effects of analgesic drugs, we recently developed a gait analysis system, which provides an index of pain severity based on walking stride disturbance. Using this system, we evaluated the therapeutic effect of topical nonsteroidal anti-inflammatory drugs (NSAIDs) in rat models of acute inflammation. We found that the gait analysis system is more sensitive than conventional evaluation methods, such as measurement of swelling or analgesia, which indicated the superiority of our system for drug screening. The approach also indicated that ketoprofen is superior to other NSAIDs for providing pain relief because of its higher skin permeability. To the best of our knowledge, this is the first report demonstrating the effectiveness of topical NSAIDs in experimental animal models of acute inflammation.

\section{Introduction}

Osteoarthritis (OA) is the most common form of arthritis and the leading cause of chronic disability [1]. Rheumatoid arthritis (RA) is a progressive autoimmune disease characterized by chronic inflammation in the limbs and joints and affects $1 \%$ of the adult population [2]. Since chronic pain caused by OA and RA negatively affects the patients' quality of life, nonsteroidal anti-inflammatory drugs (NSAIDs) are routinely prescribed for pain relief. The use of topical NSAIDs is not new in the treatment of acute and chronic pain and has been shown to provide adequate pain relief with many important advantages. These advantages include protection of the active compound from gastric enzymes, avoidance of the hepatic first-pass effect, and reduction in the risk of gastrointestinal adverse effects such as ulcer, bleeding, and perforation. Furthermore, several clinical trials in OA patients have indicated that compared to oral forms, topical formulations of NSAIDs have comparable therapeutic effects and a low incidence of adverse effects $[3,4]$.

Commonly used methods for assessing the severity of arthritis and the therapeutic effects of drugs in animals are based on the degree of inflammatory responses such as pain, edema, and analgesia in the paws and/or joints, which is subjectively determined by the investigator. The threshold for limb withdrawal or vocalization is a common parameter used to indicate pain-related behavior. However, patients with arthritis do not necessarily complain about stimulusevoked pain, but rather about pain at rest or movementinduced pain $[5,6]$. Although few methods are currently available to objectively evaluate spontaneous pain related to arthritis, gait analysis has been proven to be an objective and sensitive technique for detecting gait abnormalities in connection with pain in several arthritis models [7-9]. 
In previously reported studies, both velocity and stride length significantly decreased in arthritis models, which seems reasonable because patients with $\mathrm{OA}$ or RA often show from decreased joint utilization in association with pain, for example, when walking $[10,11]$. It has been reported that systemic administration of drugs, including NSAIDs, can improve gait disturbance in rat models of arthritis. However, as far as we know, the therapeutic effects of topical NSAID formulations have not been studied in animal models.

In this study, the clinical benefit of topical NSAIDs for pain relief in carrageenan-induced acute inflammation was assessed using our recently developed gait analysis method $[12,13]$.

\section{Materials and Methods}

2.1. Drugs and Chemicals. The following products were evaluated in this study: patch formulations including a ketoprofen patch $\left(20 \mathrm{mg} / 70 \mathrm{~cm}^{2}\right.$; Keplat, Hisamitsu UK Ltd, London, $\mathrm{UK})$, a diclofenac patch $\left(180 \mathrm{mg} / 140 \mathrm{~cm}^{2}\right.$; Flector, Bayer S.p.A, Milano, Italy), and a loxoprofen patch $\left(50 \mathrm{mg} / 70 \mathrm{~cm}^{2}\right.$; Loxonin tape, Daiichi Sankyo Co. Ltd., Tokyo, Japan) and gel formulations including a ketoprofen gel $(2.5 \% \mathrm{w} / \mathrm{w}$; Ketum, A. Menarini Pharmaceutical Ltd, Florence, Italy), a diclofenac gel $(1.16 \%$ w/w; Voltarol Pain-eze emulgel, Novartis Consumer Health, Inc., Horsham, UK), and a loxoprofen gel (1\% w/w; Loxonin gel, Daiichi Sankyo Co Ltd, Tokyo, Japan). Other chemicals were obtained from commercial sources.

2.2. Animals. Male HWY rats aged 8 weeks (Japan SLC, Inc., Shizuoka, Japan, for gait analysis and histological analysis), and male Sprague-Dawley (SD) rats aged 5 weeks (Japan SLC, Inc., for the $\gamma$-carrageenan-induced edema model, and Clea Japan, Inc., Tokyo, Japan, for the yeast-induced hyperalgesia model) were used. All animals were kept in an air-conditioned room controlled for temperature $\left(22 \pm 3^{\circ} \mathrm{C}\right)$ and humidity $(50 \% \pm 20 \%)$ under a light/dark cycle (light on from $8: 30$ to $20: 30$ ). The animals were allowed food and water ad libitum except during the experiments. All animal experiments were performed with local committee approval in accordance with Laboratory Animal Welfare guidelines.

2.3. Measurement of Gait Disturbance. Rats were habituated to the experimental apparatus, including an acrylic wheel that revolved 4.0-6.0 $\mathrm{rpm} / \mathrm{min}$ and trained to keep walking on it for $2 \mathrm{~min}$ in a day, 3 times in a week prior to experiments. On the day of the experiment, each rat was anesthetized with ether and a volume of $0.05 \mathrm{~mL}$ of $0.5 \% \gamma$-carrageenansaline solution was injected into the right knee-joint space. Walking behavior was recorded for 2 min using a DigiOn TVR (DigiOn Inc., Fukuoka, Japan). For the evaluation of therapeutic effects, each NSAID formulation was applied (patch: $20 \mathrm{~cm}^{2} /$ paw, gel: $250 \mathrm{mg} /$ paw) to the knee joint $4 \mathrm{~h}$ prior to (before administration) and/or $19 \mathrm{~h}$ after (after administration) $\gamma$-carrageenan injection for $4 \mathrm{~h}$. For the full duration of drug administration, the rats were suspended with a rodent sling cover (Lomir Biomedical Inc., Canada) to keep each drug from moving from the site of application and to reduce opportunity for oral ingestion.

Gait analysis was conducted using GAIT software (Noveltec Inc., Kobe, Japan) [12, 13]. The software automatically calculates the swing time of both hind limbs in each step cycle, and the swing time index (STI) was determined using the following formula: swing time ratio (STR) $=$ (swing time of normal hind limb)/(swing time of inflamed hind limb). When STR is greater than 1, STI $=-[(1 /$ STR $)-1]$, and when STR is less than 1 , STI $=$ STR -1 . As a single hind limb bears the weight during a swing, the swing time of one hind limb shortens if pain is experienced in the opposite hind limb, leading to a decrease in STI. The STI of each rat was calculated and averaged for $2 \mathrm{~min}$.

2.4. Measurements of Hind Paw Edema and Pain Thresholds. Edema and hyperalgesia were induced by subcutaneous injection of $1.0 \% \gamma$-carrageenan-saline solution and $20 \%$ yeast suspension, respectively, in the right hind paw [14, 15]. Each drug (patch: $7 \mathrm{~cm}^{2} /$ paw, gel: $100 \mathrm{mg} /$ paw) was topically applied to the right hind paw for $3 \mathrm{~h}$ prior to induction of inflammation. The right hind paw volume and pain thresholds were measured with a plethysmometer (MK101CMP; MUROMACHI KIKAI Co., ltd., Tokyo, Japan) and by the randall-Selitto test with analgesy-meter (MK-201D, MUROMACHI KIKAI Co., ltd.), respectively, before drug administration and $3 \mathrm{~h}$ after the induction of inflammation. In some experiments, each drug $(30 \mathrm{mg} / 0.1 \mathrm{~mL} / \mathrm{paw}$, dissolved in PBS) was mixed with yeast and directly injected into the right hind paw.

2.5. Histological Analysis. Histology of the knee joint was assessed in each of 3 control or NSAID-treated (with patch formulation of ketoprofen, diclofenac, or loxoprofen) rats. Each NSAID was applied for $4 \mathrm{~h}$ before the induction of arthritis with $0.5 \% \quad \gamma$-carrageenan-saline solution as described above. Twenty-four h later, the knee joints were removed, fixed in formalin, decalcified in $10 \%$ formic acid, embedded in paraffin, cut into $3 \mu \mathrm{m}$ sections, and stained with hematoxylin-eosin. The level of inflammatory cell inflammation was scored by a pathologist blinded to treatment conditions; the pathologist assessed the infiltration of neutrophils and mononuclear cells into the dorsal synovial membrane of the knee joint (0: no change, 1: mild, 2: moderate, and 3: severe).

2.6. In Vitro Skin Permeation Test. The cutaneous permeation profiles of NSAIDs were evaluated using hairless mouse skin and a Franz diffusion cell system. The dorsal skin was harvested from mice (Crl:SKH1-Hr ${ }^{\text {hr }}$ mice, Charles River, Yokohama, Japan) and the subcutaneous fat was removed. A piece of skin was mounted on a Franz diffusion cell, after which each NSAID solution $(50 \mathrm{mg} / \mathrm{mL}$, dissolved in acetone) was applied to the skin $\left(15 \mu \mathrm{L} / 4.9 \mathrm{~cm}^{2}\right)$. Receptor fluid (PBS, pH 7.4, $10 \mathrm{~mL}$ ) was collected every $2 \mathrm{~h}$ for $24 \mathrm{~h}$. The surface temperature of the skin was maintained at $32^{\circ} \mathrm{C}$. A $500 \mu \mathrm{L}$ aliquot of each receptor fluid specimen was mixed with $500 \mu \mathrm{L}$ of acetonitrile. After centrifugal filtration, 


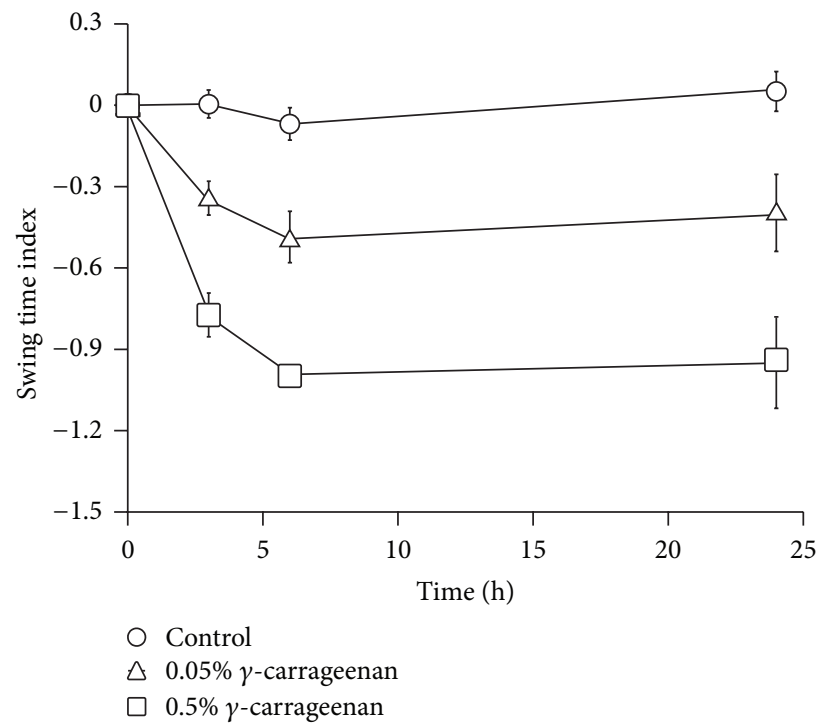

FIGURE 1: Validation of carrageenan-induced arthritis in an acute inflammation model. $\gamma$-carrageenan was injected into the hind paw, and gait disturbance was measured at 3,6, and $24 \mathrm{~h}$ after injection. Each graph represents the mean \pm SE of 4 animals.

the supernatant was used to determine the concentration of the test drug by liquid chromatography (Waters 2690, Waters Corp., Milford, MA, USA).

2.7. Statistical Analysis. The data are represented as mean \pm SE. Statistical analysis was conducted using SAS Version 6.12 (SAS Institute Japan Ltd., Tokyo, Japan). One-way ANOVA and Bartlett test, followed by the Dunnett test, were used to compare mean values among the groups. The level of significance was set at $P<0.05$.

\section{Results}

First, alterations of gait were assessed in the carrageenaninduced acute inflammation model. Carrageenan injection into the plantar surface of the hind paws decreased the STI in a dose-dependent manner, reaching the lower peak at $6 \mathrm{~h}$, which lasted for at least $18 \mathrm{~h}$ (Figure 1). Using this model, we evaluated the therapeutic effect of topical NSAIDs on walking performance. Each NSAID (ketoprofen, diclofenac, or loxoprofen), in the form of a gel or patch, was topically applied to the skin around the hind kneejoint before $\gamma$-carrageenan injection. Loxoprofen application did not show any improvement in gait, while ketoprofen dramatically improved gait disturbance (Figure 2(a)). In contrast, diclofenac slightly improved the STI, but only when the patch form was applied (Figure 2(a)). In addition to gait assessment, we also measured edema. However, a reduction in the swelling rate was barely detectable in the ketoprofen treatment group (Figure 2(b)). Furthermore, histological analysis showed that the infiltration of inflammatory cells was suppressed in the ketoprofen patch/gel treatment group, although it was not significantly different from that observed in the loxoprofen or diclofenac treatment group (Figures 3(a) and 3(b)). Hyperalgesia is another major parameter used to determine the efficacy of analgesic drugs [16]. To assess the effect of topical NSAIDs, pain threshold modulation by each agent was measured in a yeast-induced inflammation model. Pain threshold increased in all NSAID treatment groups, even in the loxoprofen treatment group that showed no STI improvement in the carrageenan model (Figure 2(c)).

These results indicate that STI is the most sensitive parameter for the assessment of topical NSAID efficacy, at least with respect to these experimental models. To further examine whether gait analysis can provide quantitative measurements of therapeutic efficacy, STI changes were measured with various doses and durations of ketoprofen patch treatments involving application of several different sizes of ketoprofen patches; then, the therapeutic outcomes were compared. As shown in Figure 4(a), gait disturbance improved in a dose-dependent manner. We also compared the pretreatment or posttreatment potency, or a combination of both. Posttreatment application improved the STI $24 \mathrm{~h}$ after carrageenan injection, as shown in Figure 2(a). However, STI was not improved when measured $4 \mathrm{~h}$ after carrageenan injection. In contrast, treatment with ketoprofen before carrageenan injection showed superior therapeutic effects compared to posttreatment ketoprofen application (Figure 4(b)). The combination treatment synergistically improved STI, especially at $24 \mathrm{~h}$ after carrageenan injection (Figure 4(b)).

The superiority of ketoprofen to other NSAIDs in terms of pain relief can be explained by 2 possible mechanisms. The first is its potentially higher permeation into the skin and the second is its potentially greater inhibitory activity on cyclooxygenase-2 (COX-2). To test the role of these mechanisms, an in vitro permeation study was conducted using hairless mouse skin. We found that the concentration of ketoprofen was the highest of all the NSAIDs tested 


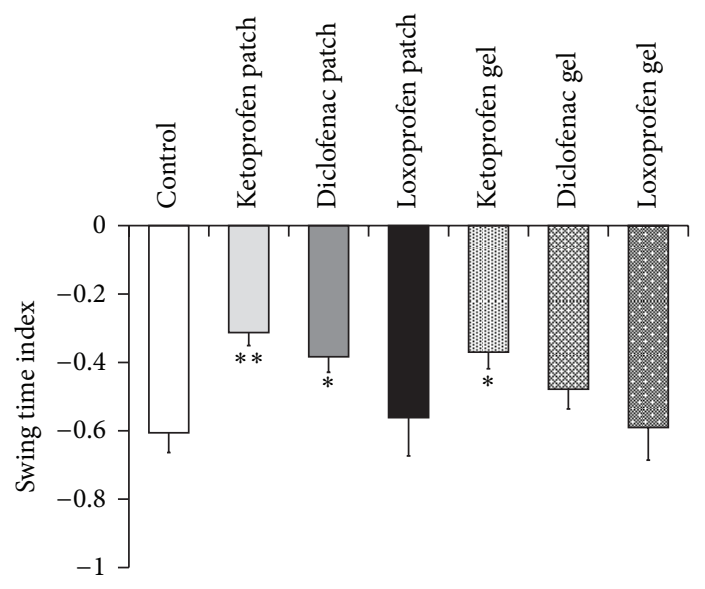

(a)

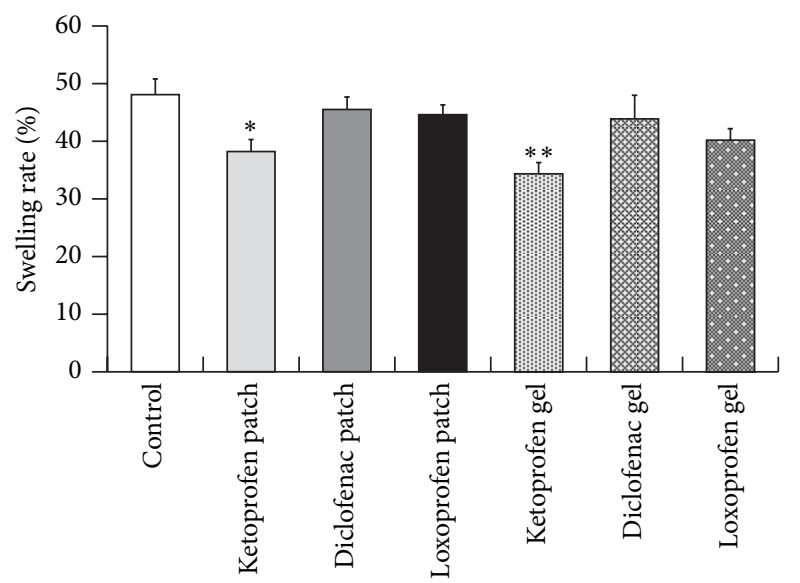

(b)

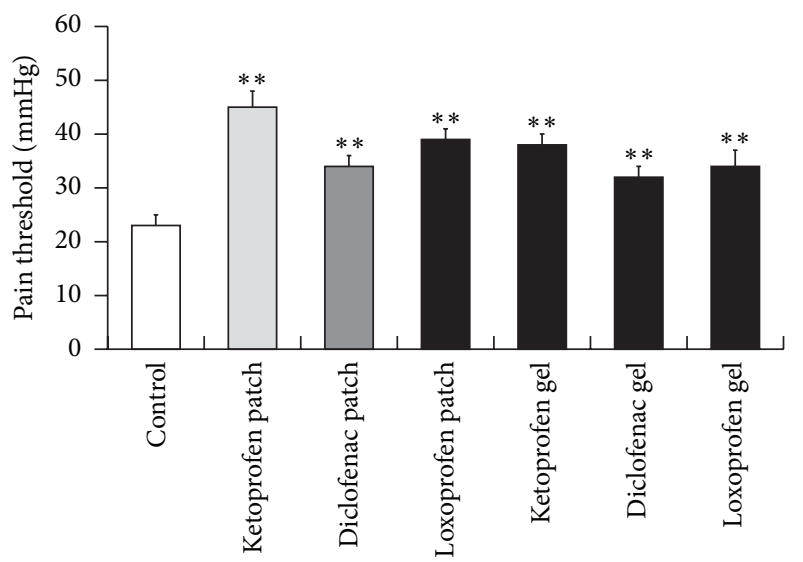

(c)

FIGURE 2: Comparison of the therapeutic effects of topical NSAID formulations in an acute inflammation model. Effects of topical NSAID formulations on gait disturbance (a) and hind paw edema (b) in a $\gamma$-carrageenan-treated inflammation model. Each column represents the mean \pm SE of 6-18 animals. Asterisks show a significant difference from control, ${ }^{*} P<0.05,{ }^{* *} P<0.01$. Hyperalgesia in yeast-treated inflammation model (c). Each column represents the mean \pm SE of 10-20 animals. Asterisks show a significant difference from control, ${ }^{*} P<0.05,{ }^{* *} P<0.01$.

at each time point, resulting in the highest cumulative amount (Figures 5(a) and 5(b)). Next, to compare the COX2 inhibition of each NSAID, identical concentrations of each agent together with yeast were directly injected into the hind paw, and pain threshold was then examined. As shown in Figure 5(c), all 3 tested NSAIDs increased the pain threshold, and the effect of ketoprofen was not superior to that of the other 2 agents.

\section{Discussion}

In this study, we showed that STI is a sensitive parameter for the measurement of the therapeutic effects of analgesic agents, and for the first time, demonstrated the efficacy of topical NSAID treatments in an experimental animal model of acute inflammation. The carrageenan-induced inflammation model is one of several universal acute inflammation models [15]. However, as shown in Figures 2 and 3, neither histological analysis nor swelling measurement was sensitive enough to detect the therapeutic efficacy of topical NSAIDs. The yeast-induced inflammation model is also used to detect changes in pain threshold [14], although this approach was also unable to demonstrate the efficacy of topical NSAID treatment. Moreover, pain threshold measurement has the potential to introduce a subjective bias depending on the experimenter. In contrast, our gait analysis system can quantitatively detect the therapeutic effects of topical analgesic agents, suggesting the relative advantage of this approach for drug evaluation (Figures 2(a), 4(a), and 4(b)).

Superior effects of topical ketoprofen were observed in walking function (swing time index, Figure 2(a)) and relief in inflammatory edema (swelling rate, Figure 2(b)), when compared to other NSAIDs used in this study. Pain threshold was significantly improved by all tested NSAIDs, and ketoprofen exerted the most potent effect (Figure 2(c)). The superiority of ketoprofen may be due to its high permeability 

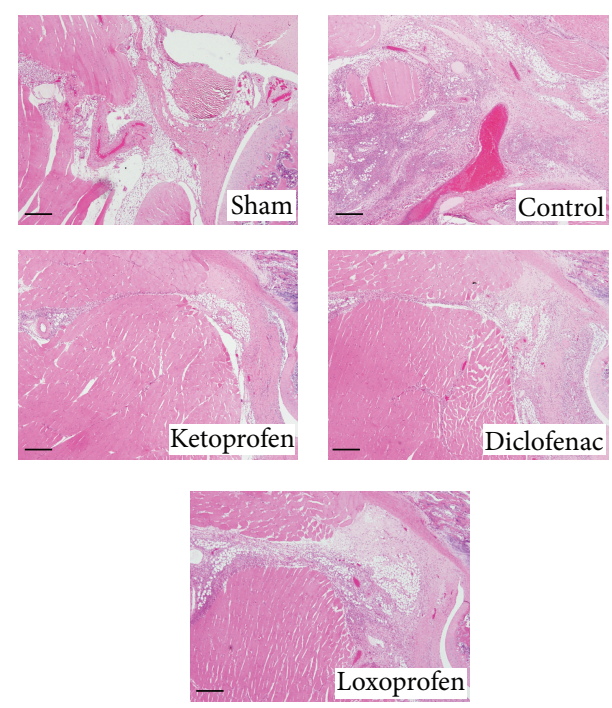

(a)

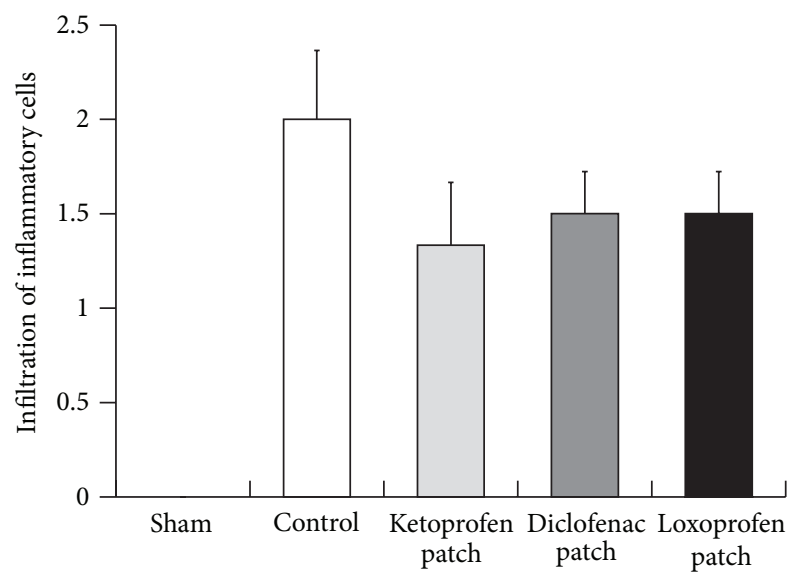

(b)

FIGURE 3: Histological analysis of knee joints of rats with $\gamma$-carrageenan-induced arthritis. Representative sections stained with hematoxylin and eosin (a). Scale bars are $300 \mu \mathrm{m}$. Histopathological scores for infiltration of neutrophils and mononuclear cells ( 0 , no change; 1 , mild; 2 , moderate; and 3, severe) (b). Each column represents the mean \pm SE of 3 animals.

Time after the $\gamma$-carrageenan injection (h)

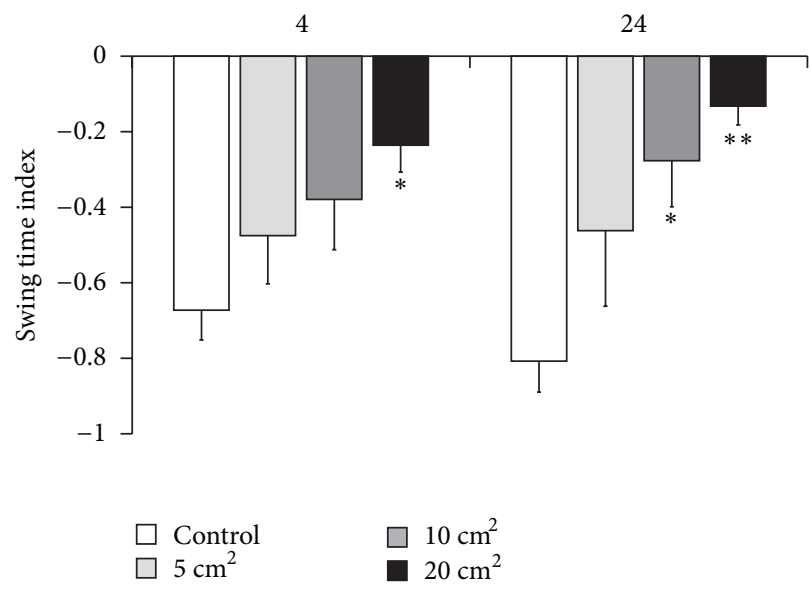

(a)

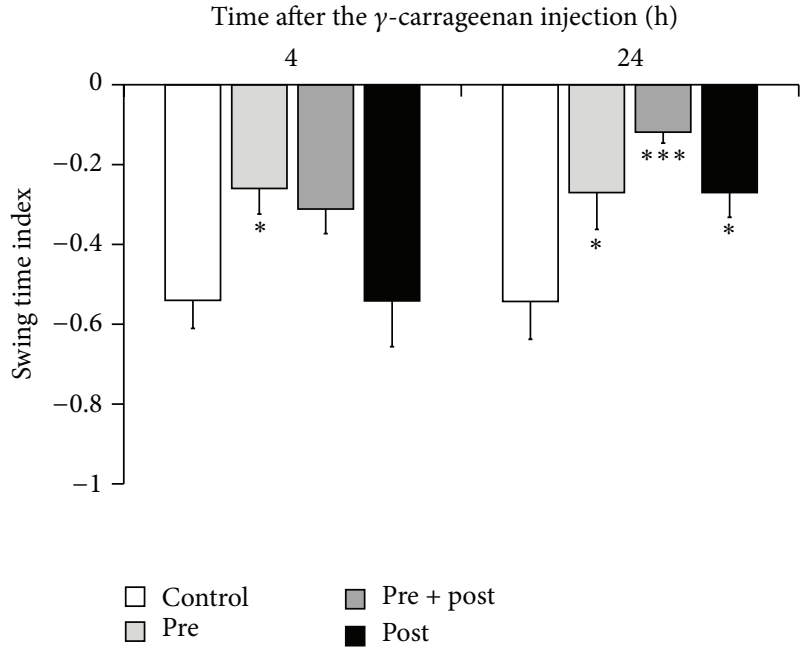

(b)

FIGURE 4: Effects of a ketoprofen patch on gait disturbance in $\gamma$-carrageenan-induced arthritis in rats. Evaluation of the timing of administration (a). The ketoprofen patch was applied on the affected knee joint before (pre), before and after (pre + post), or after (post) the induction of arthritis. Walking was evaluated with a GAIT system at 4 and $24 \mathrm{~h}$ after the injection of $\gamma$-carrageenan. A mock patch was used as a control. Each column represents the mean \pm SE of $13-14$ animals. ${ }^{*} P<0.05,{ }^{* * *} P<0.001$, compared to STI in controls. Dosedependent effects of the ketoprofen patch (b). A mock patch was used as a control. Each column represents the mean \pm SE of 7 animals. ${ }^{*} P<0.05,{ }^{* *} P<0.01$, when compared to STI in controls.

as shown in Figure 5. These results indicate ketoprofen as effective topical NSAIDs for patients with arthritis. Moreover, gait analysis can be applied to evaluate efficacy of drugs on walking function comprehensively, because it measures a physical response that directly correlates with local pain and inflammation $[14,15]$.
According to the data in Figure 4, pretreatment NSAID administration was superior to posttreatment NSAID administration, and the combination treatment was more effective than the monotherapy. In addition, STI improvement was more marked as the patch area increased (Figure 4(a)). This suggests that pain relief in clinical settings would be enhanced 


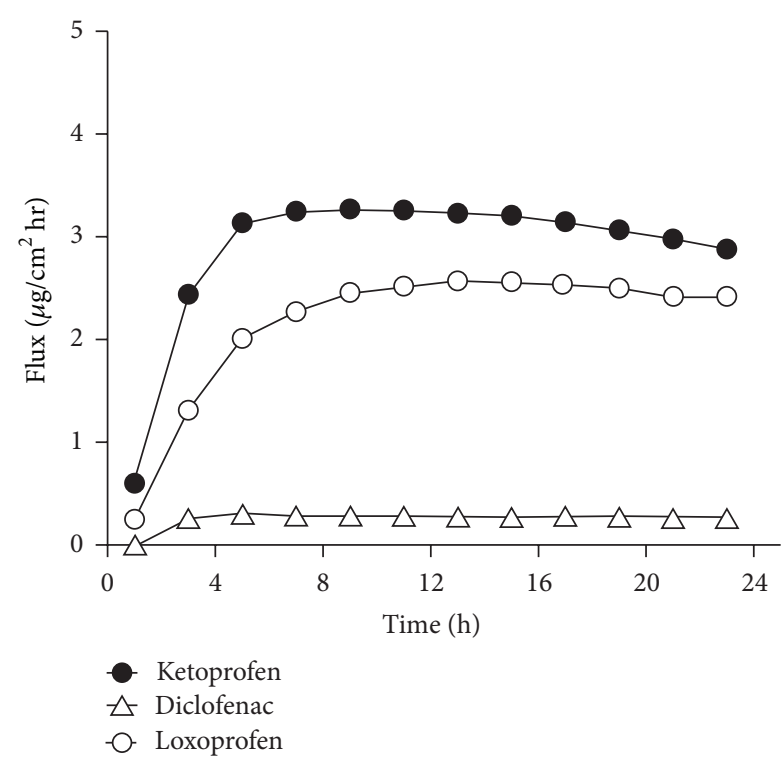

(a)

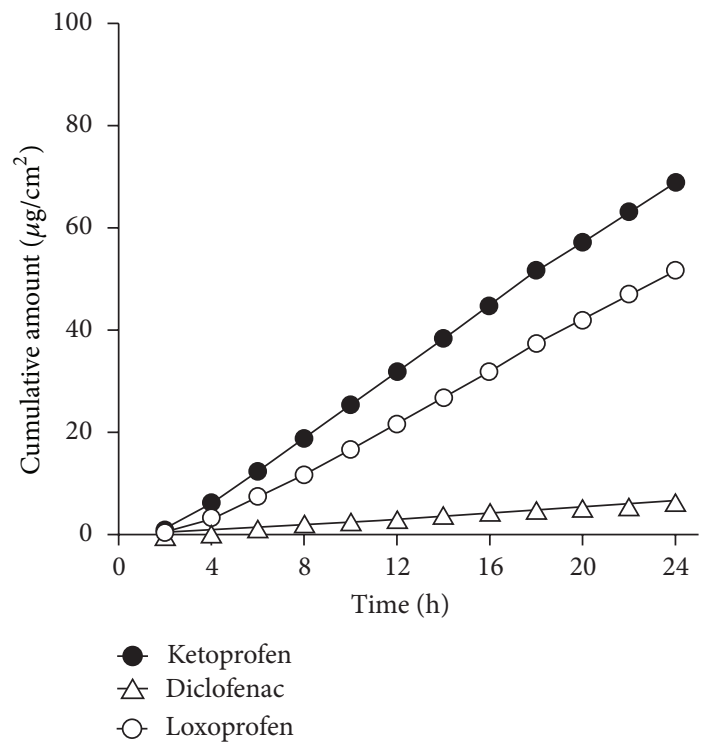

(b)

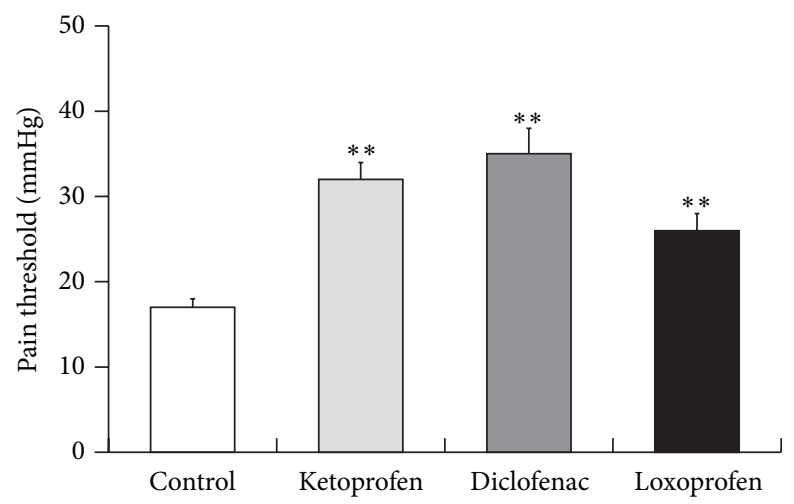

(c)

Figure 5: Advantages of ketoprofen as a topical NSAID. Cutaneous permeation profiles of multiple NSAIDs. The concentration of the test drug measured by liquid chromatography (a) and the cumulative amount of the drug calculated from the data shown in (a), (b). $N=5$ in each condition. Hind paw hyperalgesia with direct NSAID injection in the affected site (c). Each column represents the mean \pm SE of $4-5$ animals. Asterisks show a significant difference from control, ${ }^{* *} P<0.01$.

by the application of NSAIDs in as large an area as possible before walking or engaging in activities that are likely to cause pain. NSAIDs are widely used in first-line therapy for arthritis with pain [17]. Acetaminophen and weak opioids are sometimes used in combination with NSAIDs for arthritis with resistant pain. However, controlling chronic pain with oral administration of these drugs still has difficulties, as well as serious adverse effects $[18,19]$. Moreover, transudation of the orally administered NSAIDs in the synovial fluid of the patients with arthritis has been reported to be lower than that in circulation, indicating high dose administration is needed for relief pain in arthritis [20]. Thus, topical application of NSAIDs may be more useful than oral administration not only to circumvent the adverse effects but also to exert maximum effects.

In conclusion, we demonstrated the advantage of gait analysis for the detection of pain relief in an in vivo experimental model of acute inflammation. Furthermore, we showed the potential clinical utility of topical ketoprofen for the treatment of pain. We believe that these results will provide a novel strategy for drug screening.

\section{Conflict of Interests}

The authors declare no conflict of interests.

\section{References}

[1] L. Murphy and C. G. Helmick, "The impact of osteoarthritis in the United States: a population-health perspective: a population-based review of the fourth most common cause of hospitalization in U.S. adults," Orthopaedic Nursing, vol. 31, no. 2, pp. 85-91, 2012.

[2] G. S. Firestein, "Evolving concepts of rheumatoid arthritis," Nature, vol. 423, no. 6937, pp. 356-361, 2003. 
[3] L. Mason, R. A. Moore, J. E. Edwards, S. Derry, and H. J. McQuay, "Topical NSAIDs for chronic musculoskeletal pain: systematic review and meta-analysis," BMC Musculoskeletal Disorders, vol. 5, article 28, 2004.

[4] T. Massey, S. Derry, R. A. Moore, H. J. McQuay, and M. Moore, "Topical NSAIDs for acute pain in adults," Cochrane Library, no. 6, Article ID CD007402, 2010.

[5] G. La Montagna, R. Tirri, A. Baruffo, B. Preti, and S. Viaggi, "Clinical pattern of pain in rheumatoid arthritis," Clinical and Experimental Rheumatology, vol. 15, no. 5, pp. 481-485, 1997.

[6] E. Thomas, G. Peat, L. Harris, R. Wilkie, and P. R. Croft, "The prevalence of pain and pain interference in a general population of older adults: cross-sectional findings from the North Staffordshire Osteoarthritis Project (NorStOP)," Pain, vol. 110, no. 1-2, pp. 361-368, 2004.

[7] U. Heilborn, O. G. Berge, L. Arborelius, and E. Brodin, "Spontaneous nociceptive behaviour in female mice with Freund's complete adjuvant- and carrageenan-induced monoarthritis," Brain Research, vol. 1143, pp. 143-149, 2007.

[8] F. A. C. Rocha, A. G. M. Aragão Jr., R. C. Oliveira, M. M. L. Pompeu, M. R. Vale, and R. A. Ribeiro, "Periarthritis promotes gait disturbance in zymosan-induced arthritis in rats," Inflammation Research, vol. 48, no. 9, pp. 485-490, 1999.

[9] S. U. Simjee, H. Jawed, J. Quadri, and S. Saeed, "Quantitative gait analysis as a method to assess mechanical hyperalgesia modulated by disease-modifying antirheumatoid drugs in the adjuvant-induced arthritic rat," Arthritis Research and Therapy, vol. 9, no. 5, article R91, 2007.

[10] H. Schaible, M. Schmelz, and I. Tegeder, "Pathophysiology and treatment of pain in joint disease," Advanced Drug Delivery Reviews, vol. 58, no. 2, pp. 323-342, 2006.

[11] J. Viton, M. Timsit, S. Mesure, J. Massion, J. Franceschi, and A. Delarque, "Asymmetry of gait initiation in patients with unilateral knee arthritis," Archives of Physical Medicine and Rehabilitation, vol. 81, no. 2, pp. 194-200, 2000.

[12] N. Okamoto, A. Tanaka, K. Jung et al., "Silencing of into gene restores function of the ischaemic hindlimb in a rat model of peripheral arterial disease," Cardiovascular Research, vol. 92, no. 2, pp. 209-217, 2011.

[13] K. Orito, S. Kurozumi, I. Ishii, A. Tanaka, J. Sawada, and H. Matsuda, "A sensitive gait parameter for quantification of arthritis in rats," Journal of Pharmacological Sciences, vol. 103, no. 1, pp. 113-116, 2007.

[14] L. O. Randall and J. J. Selitto, "A method for measurement of analgesic activity on inflamed tissue," Archives Internationales de Pharmacodynamie et de Thérapie, vol. 111, no. 4, pp. 409-419, 1957.

[15] C. A. Winter, E. A. Risley, and G. W. Nuss, "Carrageenininduced edema in hind paw of the rat as an assay for antiiflammatory drugs," Proceedings of the Society for Experimental Biology and Medicine, vol. 111, pp. 544-547, 1962.

[16] E. E. Opas, A. Dallob, E. Herold, S. Luell, and J. L. Humes, "Pharmacological modulation of eicosanoid levels and hyperalgesia in yeast-induced inflammation," Biochemical Pharmacology, vol. 36 , no. 4, pp. 547-551, 1987.

[17] Y. C. Lee, "Effect and treatment of chronic pain in inflammatory arthritis," Current Rheumatology Reports, vol. 15, no. 1, article 300, 2013.

[18] G. Hosie and H. Bird, "The topical NSAID felbinac versus oral NSAIDs: a critical review," European Journal of Rheumatology and Inflammation, vol. 14, no. 4, pp. 21-28, 1994.
[19] L. S. Simon, L. M. Grierson, Z. Naseer, A. A. M. Bookman, and J. Z. Shainhouse, "Efficacy and safety of topical diclofenac containing dimethyl sulfoxide (DMSO) compared with those of topical placebo, DMSO vehicle and oral diclofenac for knee osteoarthritis," Pain, vol. 143, no. 3, pp. 238-245, 2009.

[20] A. Soren, "Kinetics of salicylates in blood and joint fluid," Journal of Clinical Pharmacology, vol. 15, no. 2-3, pp. 173-177, 1975. 


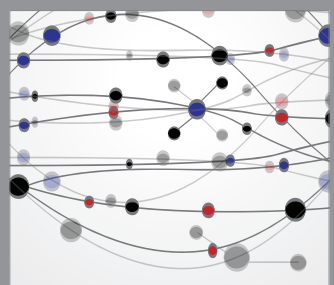

The Scientific World Journal
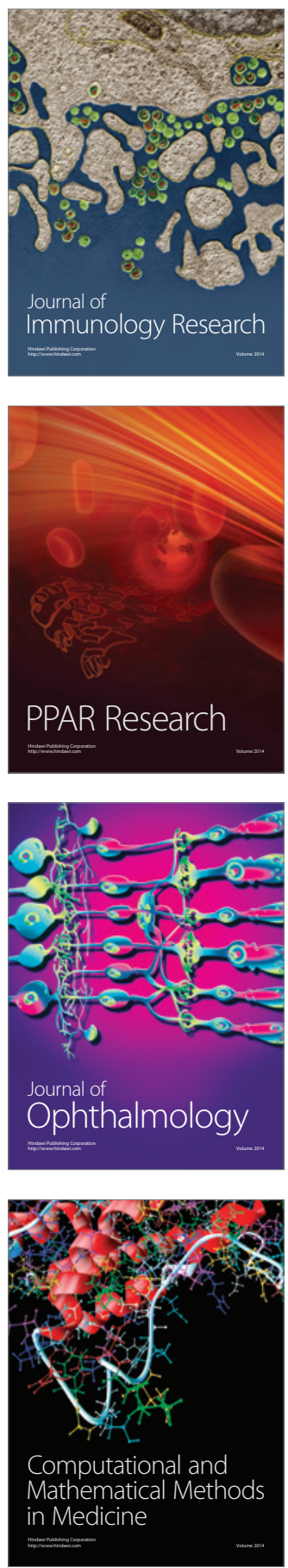

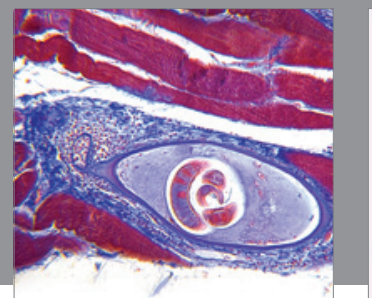

Gastroenterology

Research and Practice
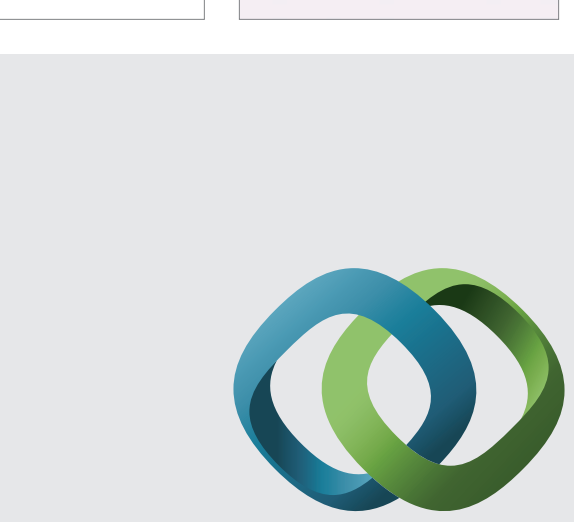

\section{Hindawi}

Submit your manuscripts at

http://www.hindawi.com
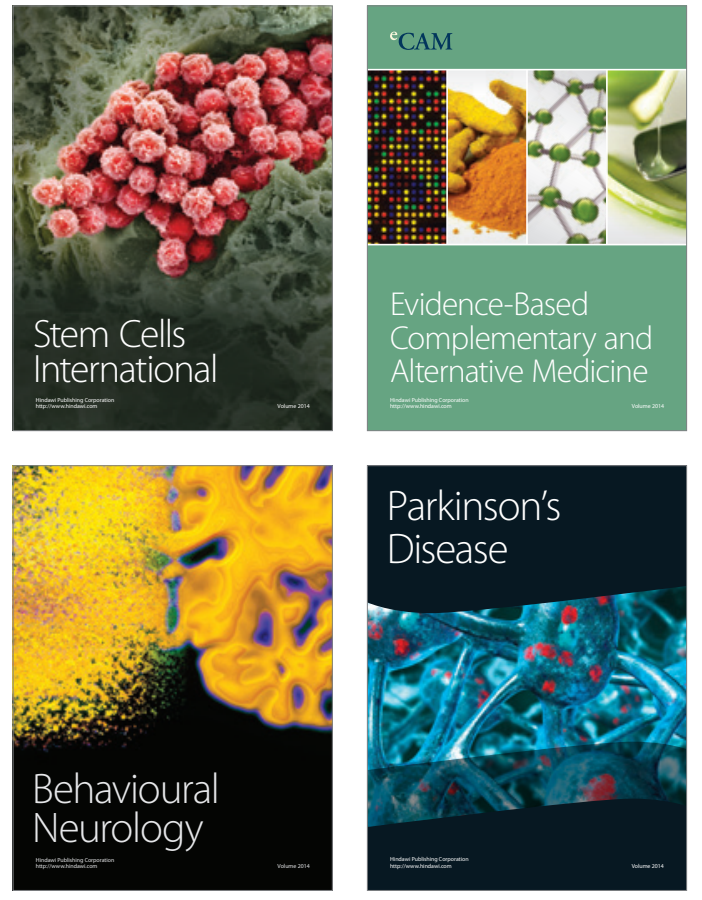
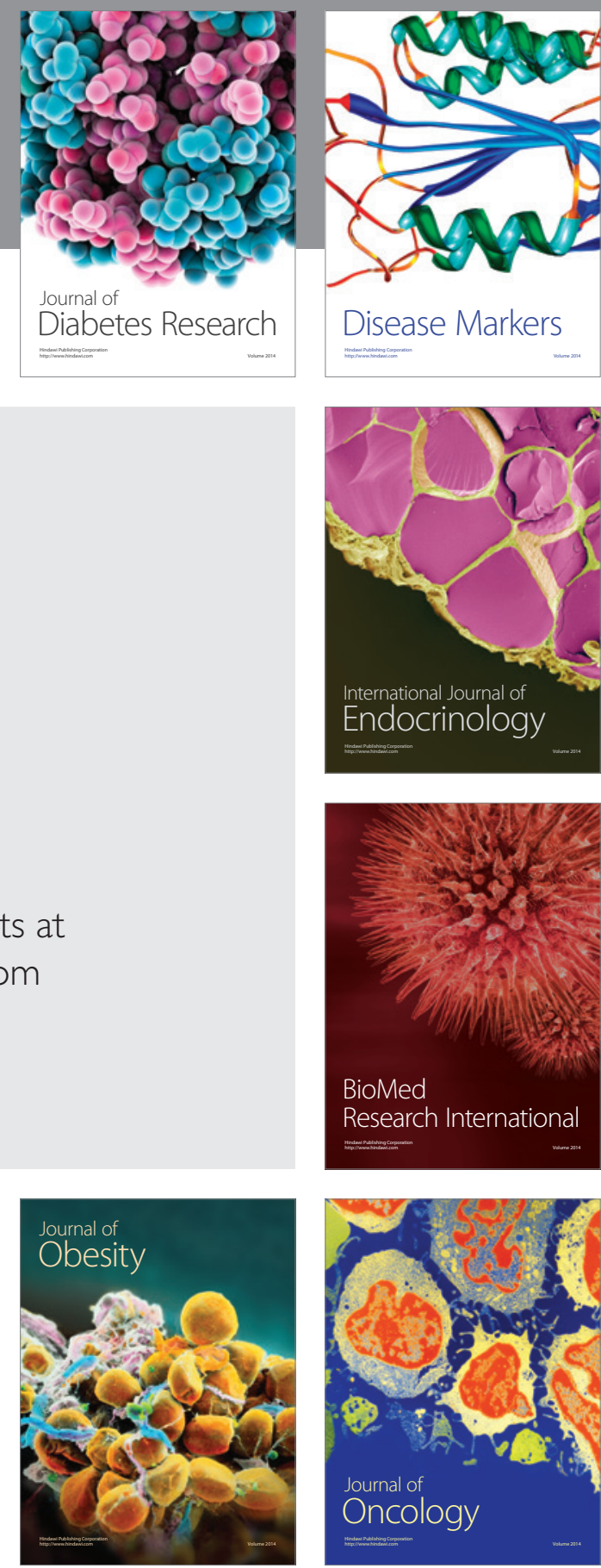

Disease Markers
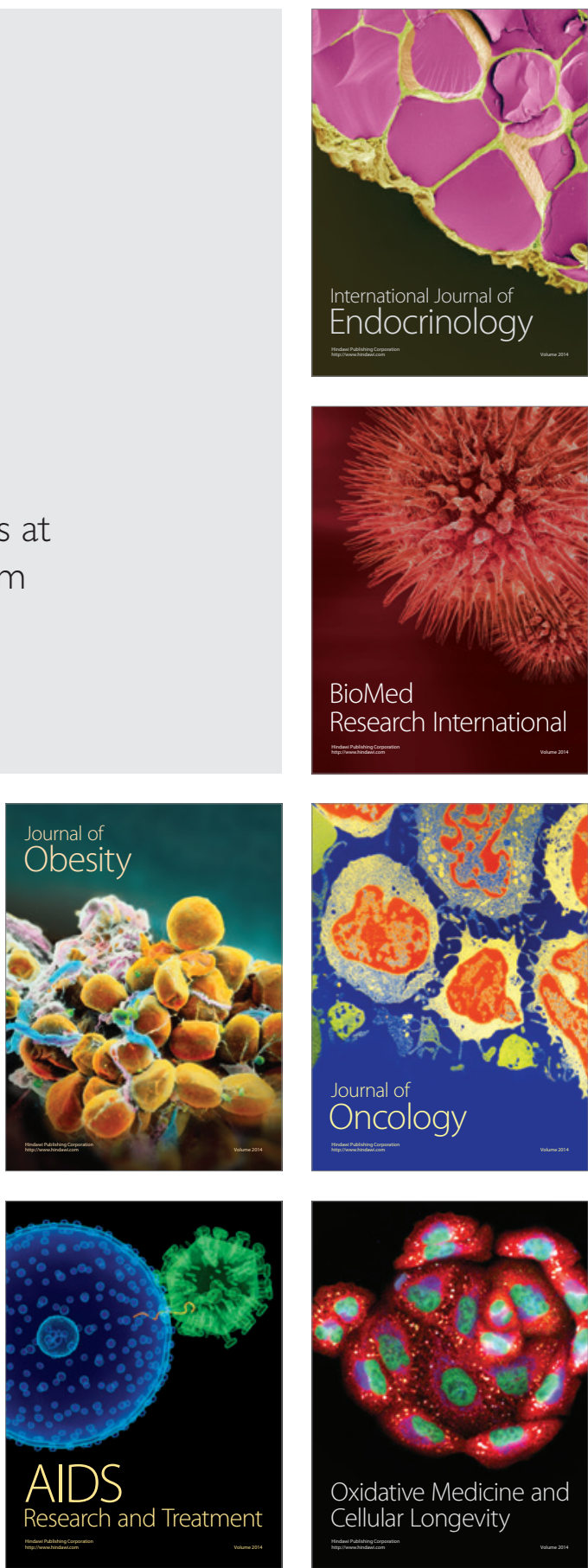